\title{
Variações espaciais na assembléia de peixes no Rio Paraíba do Sul (Barra Mansa, Barra do Piraí), Rio de Janeiro, Brasil
}

\author{
Francisco Gerson Araújo ${ }^{1}$ \\ llana Fichberg ${ }^{1}$ \\ Benjamin Carvalho Teixeira Pinto ${ }^{1}$ \\ Magna Galvão Peixoto ${ }^{1}$
}

\begin{abstract}
Spatial Variation in Fish Assemblage in the Paraíba do Sul River (Barra Mansa, Barra do Piraí), Rio de Janeiro, Brazil. The Paraíba do Sul is a major running water system in southeastern Brazil ( $\left.22^{\circ} 24^{\prime}-22^{\circ} 26^{\prime} \mathrm{S} ; 43^{\circ} 43^{\prime}-44^{\circ} 16^{\prime} \mathrm{W}\right)$, draining one of the most important industrial regions in the country. Because of its location, it has suffered a large amount of alteration due to discharges of urban and industrial effluents with direct influence in the ichthyofauna. The aim of this study is to up-to-date information on fish assemblage structure and its variation in the vicinity of a major industrial effluent outfall at Volta Redonda industrial complex. A standardized fishing sampling program, using seines, casting nets, gill nets and net trays, was carried out from October 1997 to September 1999, at six sites in three zones of the river (upstream, nearby and downstream the industrial complex), aiming to compare variation in fish assemblage due to anthropogenic alteration. Fifty-two species in 13 families were identified in the total of 7286 fish specimens. Characidae was the most diverse family contributing with $28,85 \%$ to total number of species, followed by Cichlidae (17,31\%). Overall 15,38\% of the species were introduced from other watershed systems, four exotic cichlids (genera Oreochromis Günther, 1862 and Tilapia A. Smith, 1840) and three aloctone (Cichla ocellaris Bloch \& Schneider, 1801, Hyphessobricon callistus (Boulenger, 1900) e Metynnis maculatus (Kner, 1860)). Lebistes reticulatus (Peters, 1859) was the most abundant fish species contributing to $39,7 \%$ of the total number of fish, followed by Geophagus brasiliensis (Quoy \& Gaimard, 1824) amounting to $27,4 \%$. Fish abundance was higher in the industrial zone, but only a few very dominant species were captured, while the upstream zone showed the most even community. Species replacement tolerance along the alterations gradient in the three zone seems to be a strategy to use the limited resources in the area.

KEY WORDS. Rivers, fish taxocenose, pollution, ichthyofauna, fish ecology
\end{abstract}

O rio Paraíba do Sul é um ecossistema de grande importância, pois suas águas são utilizadas, tanto para consumo doméstico e industrial, como para a produção de energia, suprindo cerca de $90 \%$ da demanda do município do Rio de Janeiro e arredores (SILVEIRA et al. 1979; BARRoso 1989). Apesar desta importância, poucas são as informações atualizadas sobre a biota, tais como as assembléias de peixes, organismos estes importantes como indicadores da qualidade ambiental do rio. O trecho mais utilizado, e portanto mais alterado do rio, situa-se entre Barra Mansa e Barra do Piraí, onde o rio atravessa o complexo industrial

1) Laboratório de Ecologia de Peixes, Universidade Federal Rural do Rio de Janeiro. Antiga Rodovia Rio - São Paulo, Km 47, 23851-970 Seropédica, Rio de Janeiro, Brasil. 
de Volta Redonda e, um pouco mais a jusante, situa-se o local de abstração da água para produção de energia pela Light (Serviços de Eletricidade S/A). Após serem utilizadas nas usinas, esta água é tratada pela CEDAE (Companhia de Águas e Esgotos do Estado do Rio de Janeiro) e distribuída para suprimento da população do município do Rio de Janeiro.

$\mathrm{O}$ aumento da atividade industrial no trecho médio-inferior do rio vem ocorrendo em ritmo acelerado, onde encontra-se implantado o segundo maior parque industrial do Estado do Rio de Janeiro. O crescente grau de poluição do rio e tributários neste trecho tem apresentado grande influência sobre a fauna e flora, uma vez que tais ambientes têm sido utilizados como via de escoamento de vários poluentes, constituindo um sério problema em relação ao equilíbrio ecológico dos recursos aquáticos (ARAÚJo 1983; TORRES et al. 1997).

Uma hipótese bastante aceita no gerenciamento de recursos aquáticos é que alterações em habitats de rios apresentam comunidades bióticas alteradas, portanto, o estudo da biota no monitoramento e avaliação de sistemas aquáticos vem alcançando grande importância na detecção de impactos de origem antrópica (LoEB 1994; SCOTT \& HALL 1997). Comunidades de peixes têm sido usadas neste sentido, pois alteram sua composição à medida que seus habitats são modificados (ANGERMEIR \& KARR 1984). A vantagem deste método é que estas comunidades integram a totalidade de fatores ambientais de um rio e, portanto, representam uma ferramenta de avaliação de qualidade rápida, econômica e compreensiva (PALLER et al. 1996).

Uma abordagem geral sobre a composição da ictiofauna da Bacia do rio Paraíba foi realizada por BRITSKI (1972) e BIZERRIL (1999). ARAÚJO (1983) relacionou as espécies da bacia no trecho entre a Represa do Funil e a barragem de Santa Cecília. ARAÚjo et al. (1995) e ARAÚJo (1996) enfocaram a composição e estrutura da comunidade de peixes do rio entre Barra do Piraí e o estuário. O objetivo deste trabalho foi realizar um diagnóstico recente da composição da ictiofauna e comparar três zonas (montante do complexo industrial, no complexo industrial e a jusante do complexo industrial de Volta Redonda), uma vez que as mudanças no ambiente poderiam estar influenciando na estrutura das populações.

\section{MATERIAL E MÉTODOS}

\section{Área de estudo}

O rio Paraíba do Sul apresenta aproximadamente $1080 \mathrm{~km}$ de extensão, banhando os Estados de São Paulo, Minas Gerais e Rio de Janeiro e compreendendo uma área de drenagem de $57.000 \mathrm{Km}^{2}$ (SIMÕES 1977). O trecho estudado, aproximadamente com $80 \mathrm{Km}$ de extensão, localiza-se no percurso médio-inferior e banha os municípios de Barra Mansa, Volta Redonda, Pinheiral e Barra do Piraí, sendo classificado como o mais poluído (PFEIFFER et al. 1986). O solo é predominantemente constituido por terrenos sedimentares de origem antiga, coberto por uma floresta tropical latifoliada, com altitude variando entre 400 e $700 \mathrm{~m}$. O clima no trecho é mesotérmico (BARBIÉRE \& KRONEMBERG 1994) com alta umidade relativa do ar, com verões quentes e chuvosos e invernos secos. 
O rio apresenta no trecho estudado uma vazão média de $318 \mathrm{~m}^{3} / \mathrm{s}$, podendo variar de $109 \mathrm{~m}^{3} / \mathrm{s}$ na estiagem a $950 \mathrm{~m}^{3} / \mathrm{s}$ em períodos de extraordinárias cheias (HYDROSCIENCE 1977). O carreamento de material alóctone para o rio é favorecido, especialmente no período das chuvas, pelo espesso manto de rochas intemperizadas, pelos declives presentes nas formações conhecidas como "meias-laranjas" e pela ausência ou má qualidade da cobertura vegetal.

\section{Métodos}

Amostragens mensais foram realizadas entre outubro de 1997 e setembro de 1999 em seis locais de coleta, distribuídos em três zonas do rio: a montante do parque industrial de Volta Redonda, no parque, e a jusante do mesmo (Fig. 1). Foram selecionados dois locais de coleta em cada zona. Os peixes foram capturados utilizando como equipamentos de pesca tarrafas, picarés e peneiras. A unidade amostral para comparação entre as zonas foi o somatório de número de peixes capturados em 15 lances de tarrafa, 20 lances de peneira e dois cercos com picaré, com as amostras tendo sido realizadas durante o dia. A rede de picaré apresentou $10 \mathrm{~m}$ de comprimento, $5 \mathrm{~mm}$ entre nós e altura de $2 \mathrm{~m}$; a peneira, diâmetro de 80 $\mathrm{cm}$ e malha de $1 \mathrm{~mm}$; e a tarrafa, diâmetro de $3 \mathrm{~m}$ e malha $2 \mathrm{~cm}$. Adicionalmente, visando o levantamento mais completo da ictiofauna, foram utilizadas redes de espera com malha de 2,5 a 5,5 cm entre-nós, comprimento de $30 \mathrm{~m}$ e altura de $2 \mathrm{~m}$, porém o material capturado não foi incluído nas unidades amostrais.

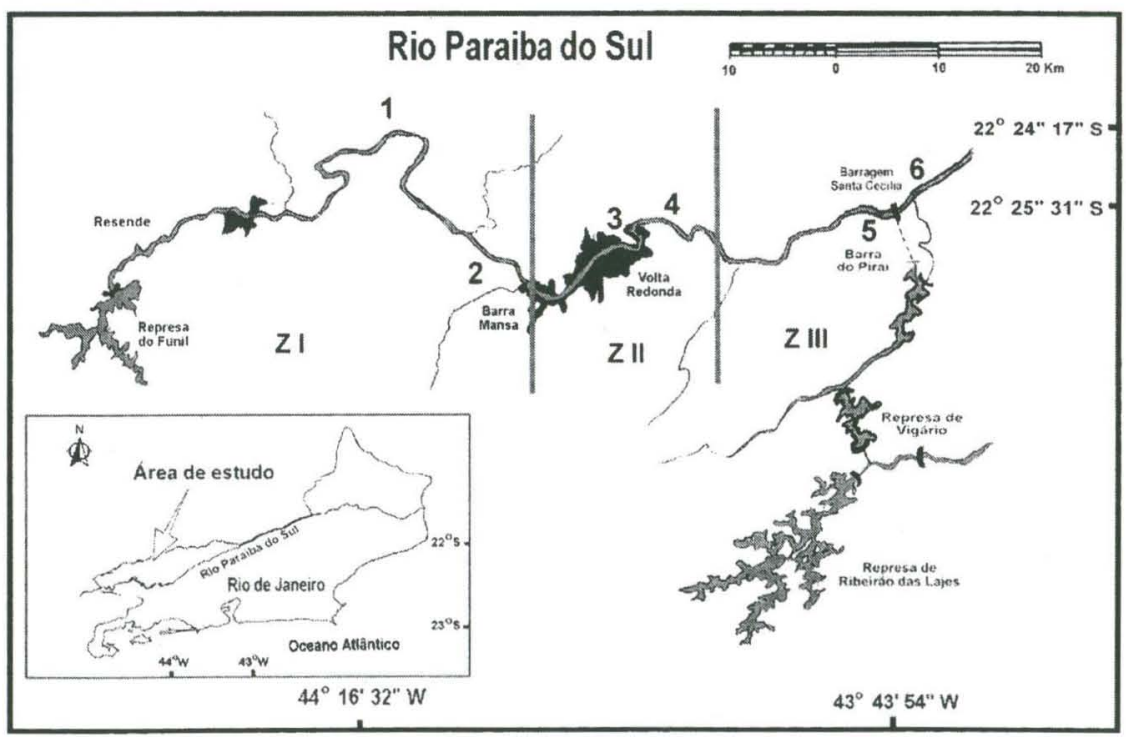

Fig. 1. Área de estudo no rio Paraíba do Sul, com indicação das três zonas amostradas (ZI) a montante do parque industrial de Volta Redonda, (ZII) parque industrial; e (ZIII) a jusante do parque industrial) e dos locais de coletas $(1,2 \ldots 6)$ em cada zona. 
O material coletado foi fixado em formol $10 \%$, posteriormente conservado em álcool $70 \%$ e formando uma coleção de referência para a região em estudo. $\mathrm{O}$ material testemunho do presente estudo ainda não foi depositado em coleção ictiológica, estando provisoriamente localizado no Laboratório de Ecologia de Peixes da Universidade Federal Rural do Rio de Janeiro. O número de peixes e de espécies por unidade amostral foi comparado entre as três zonas, meses do ano e ciclos anuais amostrados. Utilizou-se Análise de Variância (ANOVA) para comparações entre as médias e o teste de Student-Newmam-Keuls (SNK) para determinação de que médias foram significantemente diferentes ao nível de $95 \%$ de confiança, sobre os dados transformados $\left(\log _{10} \mathrm{X}+1\right)$.

Para o cálculo da similaridade ictiofaunística entre as zonas, foi utilizado o índice de Jaccard (MARGALEF 1974), expresso como $\mathrm{Sj}=\mathrm{a} /(\mathrm{a}+\mathrm{b}+\mathrm{c}$ ) onde: (a) número de espécies comuns às duas amostras; (b) número de espécies exclusivos de uma amostra; (c) número de espécies exclusivos da outra amostra.

\section{RESULTADOS}

Foram coletados um total de 7286 indivíduos distribuídos em 14 famílias e 52 espécies durante todo o período estudado. Os Ostariophysi constituíram cerca de $75 \%$ e os Acanthopterygii $25 \%$ do número de espécies, sendo o primeiro grupo constituído principalmente por Characiformes $(40,38 \%)$ e Siluriformes $(28,85 \%)$. A família de maior diversidade foi Characidae com $28,85 \%$ do número total de espécies, seguida da família Cichlidae (17,31\%).

Observou-se $15,38 \%$ de espécies introduzidas, sendo 5 espécies exóticas (provenientes de outros continentes ou de outra região biogeográfica) das famílias Cichlidae (gêneros Oreochromis Günther, 1862 e Tilapia A. Smith, 1840) e Poecilidae (Lebistes reticulatus (Peters, 1859)), e outras três espécies alóctones (provenientes de outras bacias do mesmo continente) (Cichla ocellaris Bloch \& Schneider, 1801, Hyphessobricon callistus (Boulenger, 1900) e Metynnis maculatus (Linnaeus, 1758)) (Tab. I).

As espécies mais abundantes foram Lebistes reticulatus, com $39,7 \%$ do número total de peixes capturados e Geophagus brasiliensis (Quoy \& Gaimard, 1824), com 27,4\%. Dezenove espécies (37,3\%) distribuíram-se nas três zonas de coleta e vinte e nove espécies contribuíram com menos de $1 \%$ do total de peixes, com 10 delas registradas apenas uma vez durante os dois ciclos anuais (Tab. II). Apesar do elevado número de espécies ter ocorrido nas três zonas, foram registrados alguns grupos cujas ocorrências foram restritas a apenas uma das zonas. No primeiro ciclo anual, três espécies foram exclusivas da zona I, sete da zona II e sete da zona III, enquanto no segundo ciclo anual duas espécies foram exclusivas da zona I, quatro da zona II e seis da zona III. As espécies Oreochromis sp. e Tilápia vermelha (híbrido) foram exclusivas da zona II e as espécies Corydoras nattereri e Rhamdia sp. foram exclusivas da zona III, nos dois ciclos anuais.

O número de peixes (indivíduos) variou entre as zonas, sendo significativamente maior na zona II do que nas zonas I e III (Tab. III, Fig. 2). Não foram observadas diferenças significativas na abundância de peixes entre meses e anos. 
Tabela I. Lista de espécies coletadas no rio Paraiba do Sul, trecho Barra Mansa - Barra do Pirai, em 1997/99.

\begin{tabular}{|c|c|}
\hline Espécies coletadas & Espécies coletadas (continuação) \\
\hline Ostariophysi & Rhamdia parahybae Steindachner, 1876 \\
\hline Characiformes & Rhamdella sp. \\
\hline Characidae & Callichthyidae \\
\hline Tetragonopterinae & Callichthys callichthys (Linnaeus, 1758) \\
\hline Astyanax bimaculatus (Linnaeus, 1758) & Corydoras nattereri Steindachner, 1876 \\
\hline Astyanax fasciatus parahybae Eigenmann, 1908 & Loricariidae \\
\hline Astyanax giton Eigenmann, 1908 & Hypostomus affinis (Steindachner, 1876) \\
\hline Astyanax scabripinnis (Jenyns, 1842) & Hypostomus luetkeni (Steindachner, 1876) \\
\hline Astyanax sp. & Hypostomus sp. \\
\hline Astyanax sp.2 & Loricariichthys spixii (Steindachner, 1877) \\
\hline Astyanax taeniatus (Jenyns, 1842) & Harttia loricariformes Steindachner, 1876 \\
\hline Deuterodon sp. & Rineloricaria sp. \\
\hline Deuterodon sp. 2 & Gymnotiformes \\
\hline Hyphessobricon bifasciatus Ellis, 1911 & Gymnotidae \\
\hline Hyphessobricon reticulatus Ellis, 1911 & Gymnotus cf. carapo Linnaeus, 1758 \\
\hline Hyphessobricon callistus (Boulenger, 1900) & Gymnotus sp. \\
\hline Cheirodontinae & Sternopygidae \\
\hline Probolodus heterostomus Eigenmann, 1911 & Eigenmannia virescens (Valenciennes, 1847) \\
\hline Acestrorhynchinae & Acanthopterygii \\
\hline Oligosarcus hepsetus (Cuvier, 1829) & Synbranchiformes \\
\hline Serrasalminae & Synbranchidae \\
\hline Metynnis maculatus (Kner, 1860) & Synbranchus marmoratus Bloch, 1795 \\
\hline Erythrinidae & Cyprinodontiformes \\
\hline Hoplias malabaricus (Bloch, 1794) & Poecilidae \\
\hline Hoplieritrynus unitaeniatus (Spix, 1829) & Phalloceros caudimaculatus (Hensel, 1868) \\
\hline Curimatidae & Lebistes reticulatus (Peters, 1859) \\
\hline Cyphocharax gilberti (Quoy e Gaimard, 1824) & Perciformes \\
\hline Anostomidae & Cichlidae \\
\hline Leporinus copelandii Steindachner, 1875 & Cichla ocellaris Bloch \& Schneider, 1801 \\
\hline Leporinus mormyrops Steindachner, 1875 & Crenicichla lacustris (Castelnau, 1855) \\
\hline Leporinus sp. & Crenicichla dorsocellata Haseman, 1911 \\
\hline Siluriformes & Cichlasoma facetus Jenys, 1842 \\
\hline Auchenipteridae & Geophagus brasiliensis (Quoy \& Gaimard, 1824) \\
\hline Glanidium albescens Reinhardt, 1874 & Tilapia rendalli (Boulenger, 1896) \\
\hline Parauchenipterus striatulus (Steindachner, 1876) & Oreochromis niloticus (Linnaeus, 1758) \\
\hline \multirow{2}{*}{$\begin{array}{l}\text { Pimelodidae } \\
\text { Pimelodus maculatus Lacépede, } 1803\end{array}$} & Oreochromis sp. Tilápia Vermelha - hibrido de $\mathrm{O}$. \\
\hline & $\begin{array}{l}\text { O. mossambicus (Peters, } 1844 \text { - macho) vs. } \\
\text { O. niloticus (Linnaeus, } 1758 \text { - fêmea) }\end{array}$ \\
\hline Pimelodus fur (Reinhardt, 1874) & Scianidae \\
\hline Pimelodella sp. & Pachypops adspersus (Steindachner, 1879) \\
\hline (Continua na segunda coluna) & \\
\hline
\end{tabular}

O número de espécies apresentou diferenças altamente significativas entre as zonas e os anos, com a zonas II e III tendo maior riqueza do que a zona I, e o segundo ciclo anual apresentou maior riqueza que o primeiro (Tab. III, Fig. 2). Em relação aos meses, também foram encontradas diferenças significativas na riqueza, com maiores médias em setembro-janeiro e março-abril.

O número total de peixes registrados (cumulativo) foi sempre maior na zona II, seguido da zona III, e menor na zona I, para os dois ciclos anuais (Fig. 2). Quanto ao número de espécies registradas, o mesmo padrão foi encontrado para o primeiro ciclo anual, porém no segundo ciclo anual foi maior na zona III, seguido da zona II e I. 
Tabela II. Total de peixes por zonas nos dois ciclos $\left(1 \% 2^{\circ}\right)$ anuais, de 1997 a 1999.

\begin{tabular}{|c|c|c|c|c|c|c|c|c|}
\hline \multirow{2}{*}{ Espécies } & \multicolumn{2}{|c|}{ Zona I } & \multicolumn{2}{|c|}{ Zona II } & \multicolumn{2}{|c|}{ Zona III } & \multirow{2}{*}{ Total } & \multirow{2}{*}{ Porcentagem } \\
\hline & Primeiro & Segundo & Primeiro & Segundo & Primeiro & Segundo & & \\
\hline Lebistes reticulatus & 19 & 16 & 607 & 551 & 43 & 97 & 1333 & 39,7 \\
\hline Geophagus brasiliensis & 24 & 43 & 404 & 117 & 112 & 220 & 920 & 27,4 \\
\hline Tilapia sp. & 0 & 1 & 6 & 178 & 0 & 8 & 193 & 5,7 \\
\hline Phalloceros caudimaculatus & 4 & 3 & 5 & 2 & 61 & 43 & 118 & 3,5 \\
\hline Rineloricaria sp. & 5 & 2 & 24 & 19 & 24 & 16 & 90 & 2,7 \\
\hline Astyanax giton & 7 & 2 & 8 & 36 & 12 & 21 & 86 & 2,5 \\
\hline Tilapia rendalli & 0 & 0 & 48 & 16 & 5 & 5 & 74 & 2,2 \\
\hline Astyanax sp. & 10 & 5 & 28 & 6 & 12 & 12 & 73 & 2,2 \\
\hline Astyanax bimaculatus & 4 & 31 & 0 & 5 & 10 & 15 & 65 & 1,9 \\
\hline Astyanax fasc. parahyabe & 4 & 34 & 0 & 19 & 0 & 6 & 63 & 1,9 \\
\hline Astyanax sp2. & 1 & 8 & 19 & 4 & 1 & 10 & 43 & 1,3 \\
\hline Oligosarcus hepsetus & 2 & 7 & 1 & 4 & 9 & 13 & 36 & 1,1 \\
\hline Pimelodus maculatus & 6 & 12 & 4 & 12 & 0 & 1 & 35 & 1,0 \\
\hline Deuterodon sp. & 0 & 2 & 0 & 1 & 10 & 19 & 32 & 0,9 \\
\hline Hyphessobrycon reticulatus & 0 & 0 & 0 & 0 & 0 & 24 & 24 & 0,7 \\
\hline Oreochromis niloticus & 0 & 0 & 20 & 1 & 2 & 0 & 23 & 0,7 \\
\hline Hypostomus sp. & 4 & 2 & 6 & 4 & 1 & 3 & 20 & 0,6 \\
\hline Corydoras nattereri & 0 & 0 & 0 & 0 & 8 & 11 & 19 & 0,6 \\
\hline Hyphessobricon callistus & 0 & 4 & 0 & 1 & 2 & 12 & 19 & 0,6 \\
\hline Astyanax scabripinnis & 2 & 1 & 1 & 0 & 0 & 7 & 11 & 0,3 \\
\hline Hoplias malabaricus & 1 & 5 & 2 & 1 & 1 & 1 & 11 & 0,3 \\
\hline Leporinus copelandii & 9 & 0 & 1 & 0 & 1 & 0 & 11 & 0,3 \\
\hline Callichthys callichthys & 0 & 0 & 0 & 5 & 1 & 3 & 9 & 0,3 \\
\hline Hypostomus luetkeni & 0 & 0 & 9 & 0 & 0 & 0 & 9 & 0,3 \\
\hline Hypostomus affinis & 1 & 0 & 7 & 1 & 0 & 0 & 9 & 0,3 \\
\hline Probolodus heterostomus & 4 & 4 & 0 & 0 & 0 & 1 & 9 & 0,3 \\
\hline Gymnotus cf. carapo & 0 & 2 & 1 & 0 & 0 & 1 & 4 & 0,1 \\
\hline Rhamdia parahybae & 0 & 0 & 0 & 0 & 2 & 2 & 4 & 0,1 \\
\hline Hyphessobrycon bifasciatus & 0 & 0 & 1 & 0 & 1 & 1 & 3 & 0,1 \\
\hline Tilápia vermelha & 0 & 0 & 2 & 1 & 0 & 0 & 3 & 0,1 \\
\hline Leporinus sp. & 0 & 0 & 2 & 0 & 0 & 0 & 2 & $<0,1$ \\
\hline Crenicichla dorsocellata & 1 & 0 & 0 & 0 & 0 & 0 & 1 & $<0,1$ \\
\hline Cichla ocellaris & 0 & 1 & 0 & 0 & 0 & 0 & 1 & $<0,1$ \\
\hline Cichlasoma facetus & 0 & 0 & 0 & 1 & 0 & 0 & 1 & $<0,1$ \\
\hline Crenicichla lacustris & 0 & 0 & 1 & 0 & 0 & 0 & 1 & $<0,1$ \\
\hline Cyphocharax gilberti & 0 & 0 & 0 & 0 & 0 & 1 & 1 & $<0,1$ \\
\hline Gymnotus sp. & 0 & 0 & 0 & 0 & 0 & 1 & 1 & $<0,1$ \\
\hline Hoplieritrynus unitaeniatus & 0 & 0 & 0 & 0 & 1 & 0 & 1 & $<0,1$ \\
\hline Leporinus mormyrops & 0 & 1 & 0 & 0 & 0 & 0 & 1 & $<0,1$ \\
\hline Loricariichthys spixii & 0 & 0 & 1 & 0 & 0 & 0 & 1 & $<0,1$ \\
\hline Pimelodella sp. & 0 & 0 & 0 & 0 & 1 & 0 & 1 & $<0,1$ \\
\hline Total de individuos & 108 & 186 & 1208 & 985 & 320 & 554 & 3361 & 100,0 \\
\hline Total de espécies & 18 & 21 & 24 & 22 & 22 & 27 & & \\
\hline
\end{tabular}

Foi verificada maior similaridade entre as zonas I e II (53,85\%) no primeiro ciclo de coleta, e entre as zonas I e III no segundo ciclo. De uma maneira geral, a ictiofauna entre as três zonas foi mais similar no segundo ciclo anual quando comparado com o primeiro (Tab. IV).

\section{DISCUSSÃO}

A assembléia de peixes composta de 52 espécies no trecho estudado é muito próxima da diversidade reportada no levantamento realizado por ARAÚJO (1996), que registrou 57 espécies de água doce entre Barra do Piraí e o estuário, não estando incluídos neste número as espécies estuarinas. Na compilação de FowlER (1948, 

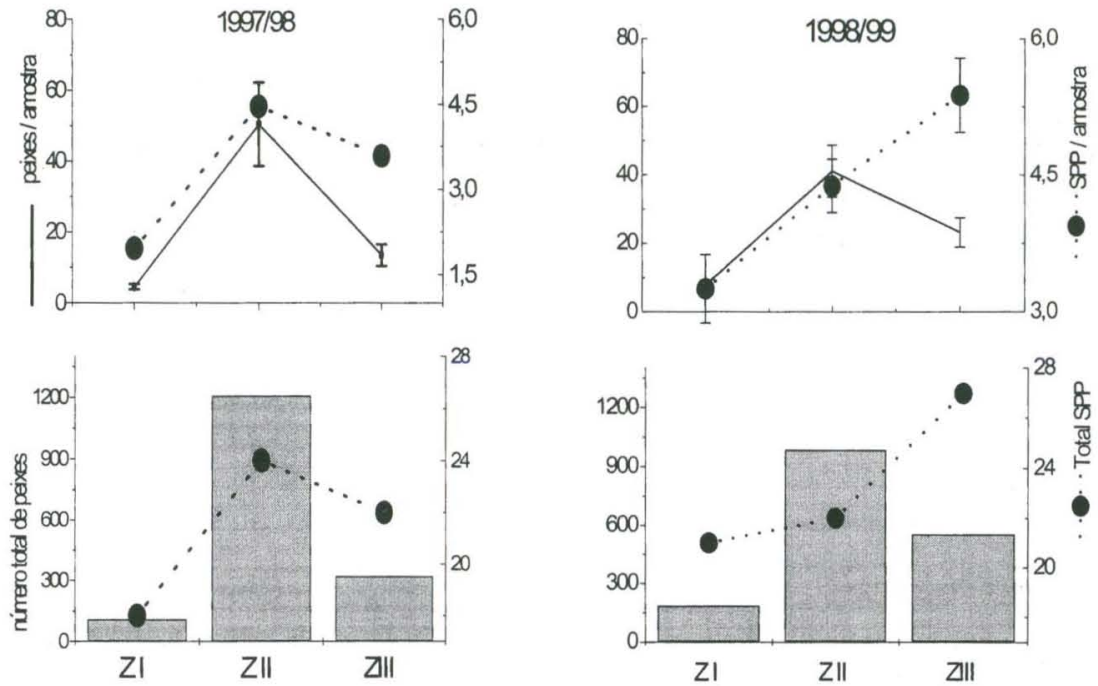

\section{Fluxo do rio}

Fig. 2. Número de espécies e número de indivíduos nas três zonas do rio Paraiba do Sul e nos dois ciclos anuais amostrados.

Tabela III. Valores de F e significâncias de ANOVA para comparações do número de indivíduos e número de espécies, entre as zonas, meses e anos. Amplitude de médias expressa entre parênteses. (am) Amostra, (spp) espécies, (ind) indivíduos.

\begin{tabular}{|c|c|c|c|c|}
\hline \multirow{2}{*}{ Fatores } & \multirow{2}{*}{ GL } & $\mathrm{F}$ & Significância & \multirow{2}{*}{ Testes SNK } \\
\hline & & Individuos & Espécies & \\
\hline Zona & 2 & $21,30 *$ & $14,6 *$ & Espécies: zona 2 e $3(4,4$ - 4,5 spp/am) >zona 1 (2,6 spp/am) \\
\hline Mês & 11 & 1,50 n.s. & 1,9 * & Individuos: zona 2 ( 45,7 ind/am) > zona 1 e $3(6,1-18,2$ ind/am) \\
\hline Ano & 1 & 0,06 n.s. & 9,6 ** & Espécies: set.jan. (3,9-4,6 spp/am); mar-abr. $(5,1-4,3$ \\
\hline Ano $\times$ més & 11 & 0,80 n.s. & 1,4 n.s. & spp/am) > mai.-ago. $(3,2-2,7$ spp/am); fev. $(3,2$ spp/am $)$ \\
\hline Ano $\times$ zona & 2 & $1,20 \mathrm{n.s}$. & 3,0 n.s. & Espécies: ano 2 ( $4,3 \mathrm{spp} / \mathrm{am})>$ ano 1 ( $3,3 \mathrm{spp} / \mathrm{am})$ \\
\hline Zona $\mathrm{x}$ mês & 22 & $0,80 \mathrm{n} . \mathrm{s}$ & 0,6 n.s. & \\
\hline Zona $\mathrm{x}$ mês $\mathrm{x}$ ano & 22 & 0,90 n.s. & 0,6 n.s. & \\
\hline
\end{tabular}

$\left(^{\star}\right)$ Diferenças significativas $(p<0,05)$; $\left(^{* \star}\right)$ diferenças altamente significativas $(p<0,01)$; (n.s) diferenças não significativas.

Tabela IV. Similaridade de Jaccard, porcentagem da ictiofauna entre as zonas nos primeiro e segundo ciclos anuais.

\begin{tabular}{cccccc}
\hline \multirow{2}{*}{ Zonas } & \multicolumn{2}{c}{ Zona I } & & \multicolumn{2}{c}{ Zona I } \\
\cline { 2 - 3 } \cline { 5 - 6 } & Primeiro ciclo anual & Segundo ciclo anual & & Primeiro ciclo anual & Segundo ciclo anual \\
\hline Zona I & 53,85 & 59,25 & & 42,86 & 65,51 \\
Zona II & & & 46,67 & 58,06 \\
\hline
\end{tabular}


1950, 1951, 1954) estão registradas 92 espécies de água doce para o rio Paraíba do Sul, sendo portanto, uma estimativa bem superior ao detectado no presente trabalho. A estimativa de uma diversidade de 130 espécies para grandes rios da Bacia do Leste, como o Paraíba do Sul feita por BizERRIL (1999) provavelmente se refere a um abrangente levantamento da fauna, onde estão incluídas as assembléias de peixes dos pequenos contribuintes, que não chegam a ocorrer no canal principal. A menor diversidade encontrada neste trabalho, além de ser um reflexo das alterações ambientais características da área estudada, também pode ser explicada pelas amostragem terem sido restritas ao canal principal do rio, notadamente em suas margens. Um exemplo disto é que não se encontra representada a família Prochilodontidae (corimbatás), bastante abundante no trecho inferior do rio, à jusante da região estudada (ARAÚJO et al. 1995), espécie característica de zona de corredeiras (LOWE-MCCONNELL 1987; BENNEMANN et al. 2000), portanto fora do alcance dos equipamentos de pesca utilizados.

A diminuição da diversidade da fauna ictiológica do rio Paraíba do Sul como resultado das alterações que o rio vêm sofrendo ao longo dos anos, foi reportado, para uma área mais ampla, por ARAÚJO (1996). O número de espécies registrado neste trabalho, onde o esforço amostral foi bastante intenso, resultado do uso de artes de pesca diferenciadas, parece corroborar esta hipótese. Este sistema aquático apresenta no curso estudado variadas fontes de alteração, destacando-se dentre elas o mau uso da terra nas áreas marginais, a extração de areia, a introdução de poluentes urbano-industriais e introdução de efluentes de indústrias químicas e siderúrgica, conforme relatado por ARAÚJo (1983), sendo tais aspectos responsáveis pela deterioração da qualidade da água.

A estrutura da comunidade de peixes das grandes bacias do leste é representada segundo (BIZERRIL 1994), por 37,7\% Characiformes, 49,2\% Siluriformes, 1,7\% Gymnotidae, 4,9\%, Cichlidae e 4,9\% de Cyprinodontiformes. A fauna nativa dos rios da Bacia do Leste é formada essencialmente por peixes Otophysi, um arranjo comum às demais bacias da região neotropical (LOWE-MCCONNELL 1987). Segundo BIZERRIL (1994, 1995), os Siluriformes apresentam maior número de espécies nos rios do leste, o que não foi observado no presente trabalho, onde os Characiformes apresentaram maior diversidade $(40,38 \%)$. Isto se deve provavelmente ao fato da área amostrada ter se restringido às margens do rio, utilizadas mais intensamente por Characiformes do que por Siluriformes, estes últimos utilizando locais mais fundos do rio. Esta separação espacial, com os Characiformes predominando nas margens e com hábitos preferencialmente diurnos (período das amostragens) e os Siluriformes concentrando-se no fundo do canal principal, com hábitos predominantemente noturnos também foi observada por BENNEMANN et al. (2000).

A comunidade foi dominada por duas espécies omnívoras (Lebistes reticulatus e G. brasiliensis), principalmente na zona II, a área mais impactada do trecho estudado. Segundo ODUM (1969), quando os recursos naturais são alterados, as espécies especialistas são substituídas pelas oportunistas. ROUGHARDEN (1974) concorda com tal hipótese, quando afirma que peixes generalistas apresentam maior vantagem sobre os peixes especialistas, o que também foi mencionado por KREBS (1985), que afirma que grande maioria das espécies tem pequena abundância, 
provavelmente, devido a limitada disponibilidade de alimento ou por não suportar a baixa qualidade da água causada por alterações antrópicas. Mais de um terço das espécies não parecem sofrer limitações em sua distribuição causadas, pelo impacto na zona II, pois embora em pequena abundância relativa, ocorrem em todas as três zonas. Isto é uma indicação de que o ambiente embora bastante alterado pela presença de poluentes, os quais foram encontrados em elevadas concentrações em alguns componentes da taxocenose de peixes (ARAÚJO 1983), não é fator limitante na ocorrência das espécies, porém provavelmente concorre para a relativamente baixa abundância registrada.

Não obstante a ampla distribuição de um grande número de espécies, também observou-se considerável ocorrência de determinados grupos exclusivos de apenas uma das zonas, sugerindo restrições no uso de determinadas áreas. Um maior número de espécies foi exclusivo da zona III e um menor, da zona I, enquanto na Zona II somente as tilápias, conhecidas por sua elevada resistência à condições ambientais adversas, foram consistentemente exclusivas nos dois ciclos anuais. Este padrão de substituição de espécies parece sugerir uma estratégia de utilização dos recursos limitados, onde os peixes vêm mantendo uma diversidade semelhante entre as três zonas, independente do nível de alteração. A partilha dos recursos, neste caso, parece ser interpretada como a ocupação de nichos tróficos mais alterados por espécies mais generalistas, oportunistas no seu trecho crítico (Zona II), e por outras espécies mais especializadas, nas zonas de menor influência antrópica.

A introdução de espécies alóctones pode deslocar ou reduzir drasticamente as populações autóctones. Tais espécies introduzidas também podem alterar o ambiente, pela introdução de organismos patológicos capazes de infestar as espécies autóctones, as quais normalmente não possuem mecanismos de defesa contra tais organismos. Acrescenta-se a isto, o fato de algumas das espécies introduzidas serem carnívoras predadoras, como o tucunaré - Cichla ocellaris, ocupando, portanto, o topo da cadeia alimentar e alterando de maneira mais abrangente a estrutura da comunidade. Grande parte destas espécies são provenientes de atividades de cultivo e de recreação, tipo pesque-e-pague, localizadas nas proximidades do rio, notadamente nas zonas II e III, onde foi encontrado maior número de espécies.

AGRADECIMENTOS. Agradecemos ao Setor de Ictiologia do Museu Nacional, através do Dr. Paulo A. Buckup e do Mestrando Filipe Augusto G. de Melo, pelo auxílio na identificação dos Characiformes. Agradecemos à Companhia Siderúrgica Nacional - CSN, pelo suporte financeiro, através de contrato de serviços com a Universidade Federal Rural do Rio de Janeiro, o que tornou possível a realização deste trabalho.

\section{REFERÊNCIAS BIBLIOGRÁFICAS}

ANGERMEIER, P.L. \& J.R. KARR. 1984. Relationships between woody debris and fish habitat in a small warmwater stream. Trans. Amer. Fish. Soc. (113): 716-726.

ARAújo, J.R.S. 1983. Projeto de biodetecção de tóxicos em sistemas fluviais de utilização em captação de água para sistemas públicos de abastecimento. Cadernos FEEMA, Série Congressos, Rio de Janeiro, $17 / 83$ (DEPEA/DNH).

Araúso, F.G. 1996. Composição e estrutura da comunidade de peixes do médio e baixo rio Paraíba do Sul, RJ. Rev. Brasil. Biol. 56 (1): 111-126. 
ARAúJo, F.G.; A.C.A. SAntos \& M.R.F. Simoni. 1995. Variação espacial e temporal da comunidade de peixes do Médio e baixo rio Paraíba do Sul, RJ. Rev. Univ. Rural. Sér. Ciênc. da Vida, Rio de Janeiro, 17 (2) 61-73.

BarbiÉre, E.B. \& D.M.P. Kronembeg. 1994. Climatologia do litoral sul-sudeste do Estado do Rio de Janeiro. Cadernos de Geociências, Rio de Janeiro, 12: 57-73.

Barroso, L.V. 1989. Diagnóstico Ambiental para a Pesca de Águas Interiores no Estado do Rio de Janeiro. Rio de Janeiro, IBAMA-Doc.ACUMEP, $n^{\circ} 4,177 \mathrm{p}$.

Bennemann, S.T.; O.A. Shibatta \& J.C. Garavello. 2000. Peixes do rio Tabagi- uma abordagem ecológica. Londrina, Ed. Univ. de Londrina, 62p.

BizerRIL, C.R.S.F. 1994. Análise taxonômica e biogeográfica da ictiofauna de água doce do leste brasileiro. Acta Biológica Leopoldensia, São Leopoldo, 16 (1): 51-80.

- 1995. Estrutura quantitativa de comunidades de peixes em um rio costeiro do sudeste brasileiro. Acta Biológica Leopoldensia, São Leopoldo, 17 (2): 57-80.

1999. A Ictiofauna da Bacia do Rio Paraíba do Sul. Biodiversidade e Padrões Biogeográficos. Brazil. Arch. Biol. Technol., Curitiba, 42 (2): p. 233-250.

BrITSKI, H.A. 1972. Peixes de água doce do estado de São Paulo-Sistemática, p. 79-108. In: ComisSÃo Interestadual da Bacia do Parana-Urugual (Ed.). Poluição e piscicultura. São Paulo, Faculdade de Saúde Pública USP, Instituto de pesca, 216p.

- 1992. Conhecimento atual das relações filogenéticas de peixes neotropicais, 42-57. In: Situação e perspectivas da ictiologia no Brasil. Maringá, Fund. Univ. Est. Maringá, Nupelia, SBI, $127 \mathrm{p}$.

Fowler, H.W. 1948, 1950, 1951. Os peixes de água doce do Brasil. I. Arquivos Zool., São Paulo, 6: $1-204,205-404,405-625$.

—. 1954. Os peixes de água doce do Brasil. II. Arquivos Zool., São Paulo, 9: 1-400.

HydrosCIENCE INC. 1977. The Paraíba do Sul river water quality study. BRA-73/003. New Jersey, Technical Report 6, 254p.

LOEB, S.L. 1994. An ecological context for biological monitoring, p. 3-7. In: S.L. LoEB \& A. SPACIE

(Ed.). Biological monitoring of aquatic systems. Boca Raton, Lewis Publistiers, 381p.

Lowe-McConnelt, R.H. 1987. Ecological Studies in tropical fish communities. Cambridge, Univ. Press, $382 \mathrm{p}$.

KREBS, C.J. 1985. Ecology. Harper \& Row, New York, 800p.

Margalef, R. 1974. Ecologia. Barcelona, Ed. Omega, 951 p.

OduM, E.P. 1969. Ecologia. São Paulo, Pioneira, 201p.

Paller, M.H. 1996. Use of Fish Communities to Assess Environmental Impacts in South Carolina Coastal Plain Streams. Trans. Amer. Fish. Soc. 125: (5): 633-644.

Pfeiffer, W.C.; M. Fiszman; O. Malm \& J.M. Azcue. 1986. Heavy metal pollution in the Paraiba do Sul River, Brazil. Sci. Total Environ., Amsterdã, 58: 73-79,

ROUGHGARDEN, J. 1974. Species packing and the competition function with illustrations from coral reef fish. Theoretical Pop. Biol., San Diego, 5: 163-186.

SCOTT, M.C. \& L.W. HALL JR. 1997. Fish Assemblages as Indicators of Environmental Degradation in Maryland Coastal Plain Streams. Trans. Amer. Fish. Soc. 126 (3): 349-359.

Silveira, R.S.A; D.H. Negreiros; R. Lozinsky; F.A. Almeida; G.M. Wajnstajn \& L.C.K. Oliveira. 1979. Estudo da Bacia do Rio Paraíba do Sul (Trecho Funil - Sta. Cecília - Guandu). Cadernos FEEMA, Série Técnica, Rio de Janeiro, 9 (79): 1-64p.

SIMÕES, D.F. 1977. Paraiba - o rio da sobrevivência. Saneamento, Rio de Janeiro, 51 (1/2): 8-18.

TORRES, J.P.M.; E.D.R. VIEIRA; G.F. KoOPMANS \& J. JAPENGA. 1997. The fate of organic micropollutants in a tropical ecosystem: The Paraiba do Sul-Guandu (PSR-GR) river system case study, p. 47-53. In: O. MALM \& J. JAPENGA (Eds). International workshop on organic micropollutants in the environment. Rio de Janeiro, 250p.

Recebido em 15.VI.2000; aceito em 23.V.2001. 